\title{
Estandarización de un Método Cromatográfico para la Identificación del ácido Giberélico en Semillas de Maíz (Zea mays L.)
}

\section{Standardization of a Chromatographic Method for the Identification of Giberelic Acid in Corn Seeds (Zea mays L.)}

Juan David Rivera ${ }^{\mathrm{a}, \mathrm{b} *}$ Aceptación: 20 de agosto de 2017

\author{
Yaned Milena Correa ${ }^{\mathrm{a}, \mathrm{b}, \mathrm{c}}$ \\ Diana Marcela Ocampo ${ }^{b}$
}

\section{Resumen}

El maíz (Zea mays L.) es uno de los cereales de mayor importancia a nivel mundial por sus múltiples aplicaciones industriales; además, por tener tiempos de germinación cortos, es útil en diversos procesos experimentales. En la búsqueda de nuevas alternativas que aceleren los procesos de germinación de semillas, la estimulación de las fitohormonas es un mecanismo que cada vez presenta más estudios en la literatura con el fin de aumentar la productividad en el sector agrícola en productos de alto consumo como lo son los cereales. Dentro de estas moléculas, el ácido giberélico ha sido empleado de diversas maneras para promover estos procesos. Por esta razón, se hace necesario la implementación de técnicas analíticas que permitan identificar y cuantificar esta fitohormona de manera rápida y eficaz. En este estudio se realizó la estandarización de un método por cromatografía líquida de alta eficiencia con arreglo de diodos para la identificación del ácido giberélico en semillas de maíz germinadas. Las mejores condiciones encontradas para el analito en estudio fueron: fase estacionaria reversa, fase móvil en gradiente de acetonitrilo-ácido fórmico $0.01 \%$, flujo de $0.5 \mathrm{~mL} \mathrm{~min}^{-1}$, tiempo de corrida de $45.0 \mathrm{~min}$. y longitud de onda de análisis $195 \mathrm{~nm}$. Bajo estos parámetros el ácido giberélico tuvo un tiempo de retención de $16.670 \pm 0.050 \mathrm{~min}$. Estos resultados permitieron la identificación apropiada de esta fitohormona y se considera que las condiciones encontradas en este trabajo, podrían adaptarse para la determinación de este analito en otras matrices.

Palabras clave: fitohormonas, germinación, HPLC, cereales

a Grupo de Investigación en Campos Electromagnéticos Medio Ambiente y Salud Pública. Facultad de Ciencias Exactas y Naturales. Universidad de Caldas. Sede Principal. Calle 65 N 26-10. Manizales,

* Autor de correspondencia: juan.rivera@ucaldas.edu.co

b Grupo de Investigación en Estudios Ambientales en Agua y Suelo. Facultad de Ciencias Exactas y Naturales. Universidad de Caldas. Sede Principal, calle $65 \mathrm{~N}^{\circ}$ 26-10. Manizales, Colombia.

c Grupo de Investigación en Cromatografía y Técnicas Afines (GICTA). Facultad de Ciencias Exactas y Naturales. Universidad de Caldas. Sede Principal, calle 65 N²6-10. Manizales, Colombia. 


\begin{abstract}
Corn (Zea mays L.) is one of the most important cereal worldwide, because of its multiple industrial applications; besides, by having short germination times, it is useful in experimental processes. In the search of new alternatives that fast up seed germination processes, phytohormone stimulation is a mechanism that increasingly presents more studies in the literature in order to increase productivity in the agricultural sector in high consumption products such as son cereals. Inside these molecules, gibberellic acid is used in several ways to promote these processes. This is why it is necessary to implement analytic techniques that allow the identification and quantification of this phytohormone in a fast and efficient way. In this study, standardization of a method through high performance liquid chromatography with diode array detection for identification of giberellic acid in germinated corn seeds, was made. The best found conditions for the studied analyte were: stationary phase reversed, mobile phase in gradient of acetonitrile formic acid $0.01 \%$, flux $0.5 \mathrm{~mL} \mathrm{~min}^{-1}$, running time $45.0 \mathrm{~min}$. and analysis wavelength $195 \mathrm{~nm}$. Under these parameters, gibberellic acid had retention time of $16.670 \pm 0.050$ min. These results allowed the proper identification of this hormone and it is considered that they could be adapted to determine this analyte in other arrays.
\end{abstract}

Keywords: Phytohormones, germination, HPLC, cereals. 


\section{INTRODUCCIÓN}

El maíz (Zea mays L.) es de gran importancia económica a nivel mundial; toda la planta puede ser aprovechada y las aplicaciones posibles de este cereal incluyen alimento, forraje o materia prima para diferentes industrias [1].

Como alimento, se puede utilizar todo el grano, maduro o no, o se pueden emplear técnicas de molienda en seco para obtener productos intermedios. También a partir de él puede producirse almidón, aceite, proteínas, bebidas alcohólicas y combustible [2]. Globalmente el maíz se cultiva en más de 140 millones de hectáreas con una producción anual de más de 580 millones de toneladas métricas, lo que lo convierte en el segundo cultivo más importante del mundo después del trigo [1]. A nivel experimental el maíz es de gran importancia dado sus cortos tiempos de germinación y a que puede ser utilizado como indicador en los procesos germinativos de la semilla. La germinación del maíz implica indispensablemente la presencia del ácido giberélico $\left(\mathrm{GA}_{3}\right)$, debido a que esta hormona induce la producción de enzimas hidrolíticas como las $\alpha$-amilasas, las cuales desencadenan procesos metabólicos en la planta generando su crecimiento y desarrollo [3]. El $\mathrm{GA}_{3}$ es una fitohormona que hace parte de la familia de los diterpenos tetracíclicos de naturaleza ácida [4], la cual es esencial para el desarrollo de las plantas; está encargada de controlar aspectos relacionados con su desarrollo y crecimiento, especialmente la germinación. El $\mathrm{GA}_{3}$ ha sido evaluado en diferentes especies vegetales tales como Arabidopsis thaliana [5], fresno blanco (Fraxinus americana) [6], arroz (Oryza sativa) [7] y flores de melocotón (Prunus persica L.) [8], y se ha utilizado de diferentes maneras para acelerar procesos de germinación [9]. Sin embargo, los trabajos reportados sobre esta fitohormona en el maíz se centran en el papel fisiológico en los procesos de germinación. Soló el trabajo realizado por Rivera et al., 2017 [10] reporta cuantificación de ácido giberélico en semillas de maíz con el fin determinar el mejor método de extracción de esta fitohormona.

Los bioensayos han sido utilizados como una técnica para detectar el ácido giberélico en extractos parcialmente purificados. Sin embargo, el uso de estos protocolos se ha dejado de lado a medida que se han desarrollado técnicas físicas de medida que permiten la identificación y la cuantificación precisa de giberelinas específicas a partir de pequeñas cantidades de tejido vegetal (del orden de mg a g) [11]. En la actualidad se emplea con más frecuencia la cromatografía líquida de alta eficiencia con detector de arreglo de diodos (HPLC-DAD) [12]-[14] y la cromatografía líquida de alta eficiencia acoplada a espectrometría de masas (HPLC-MS) [5], [7], [15], Estas técnicas tienen como ventajas mayor eficiencia en la separación y alta especificidad en la detección del analito de estudio [16]. Otro método menos utilizado es la electroforesis capilar acoplada a espectrometría de masas (CE-MS) [17] y la cromatografía de gases acoplada a espectrometría de masas (GCMS) [18]-[20], sin embargo, en esta última técnica cromatográfica la muestra a analizar debe ser derivatizada previamente lo cual alarga el proceso de análisis y no asegura la modificación de la muestra [21].

Partiendo de lo anterior, el objetivo de este trabajo fue estandarizar un método cromatográfico para la identificación del ácido giberélico a partir de semillas germinadas de maíz (Zea mays L.), el cual permitirá hacer evaluaciones de este analito en diferentes matrices vegetales, en menor tiempo y costo. 


\section{MATERIALES Y MÉTODOS}

\section{Reactivos químicos}

El estándar del ácido giberélico empleado fue Sigma Aldrich (Sigma, USA), el acetonitrilo y el metanol fueron grado cromatográfico (Fisher Scientific, USA), el acetato de etilo $\mathrm{y}$ el ácido fórmico fueron grado analítico (Scharlau, España). El agua fue purificada empleando un sistema Direct-Q (Millipore, USA).

\section{Muestra de estudio}

Se usaron semillas de maíz (Zea mays L.) variedad ICA-V305 tipo comercial producidas por la empresa Semillas del Pacífico (Cartago, Colombia). Se seleccionaron semillas sin daños visibles y con forma uniforme, las cuales tuvieron una masa promedio de 0.3878 $\pm 0.0002 \mathrm{~g}$. y un volumen promedio de 0.356 $\pm 0.008 \mathrm{~cm}^{3}$.

\section{Preparación de la muestra}

Las semillas de maíz previamente seleccionadas se colocaron en cajas Petri (100 x 15) mm., con papel absorbente como soporte. A cada caja se le adicionaron 12.0 $\mathrm{mL}$ de agua destilada y se colocaron en una incubadora Incucell de $222 \mathrm{~L}$ sin luz, a una temperatura de $30.1 \pm 0.1{ }^{\circ} \mathrm{C}$. La humedad del sistema fue de $59.00 \pm 3.39 \%$. Después de 48 horas, las semillas se sacaron de la incubadora y fueron trituradas en un molino eléctrico, hasta obtener un polvo muy fino que se guardó en bolsa de sello hermético y en un congelador (Thermo Scientific) a -20 ${ }^{\circ} \mathrm{C}$ para su posterior extracción [22].

\section{Extracción del ácido giberélico}

La obtención del $\mathrm{GA}_{3}$ se realizó empleando la técnica de extracción dinámica con disolvente asistida por sonicación (DSASE), según el método descrito por [10], en el cual se utilizó como solvente acetonitrilo - ácido fórmico $5 \%$ (80:20). La celda de extracción de acero inoxidable de $3.0 \mathrm{~mL}$. se llenó con $0,8000 \mathrm{~g}$. de muestra y se colocó en el interior de un baño ultrasonido (Branson 5210) al cual se le controló la temperatura del agua para que no fuera superior a $35.0^{\circ} \mathrm{C}$. Posteriormente el sistema se conectó a una bomba peristáltica para controlar el flujo del solvente a través de la celda, siendo este de $0.4 \mathrm{~mL} \mathrm{~min}^{-1}$; la extracción se realizó por un tiempo $25.0 \mathrm{~min}$., al final del cual se obtuvo un volumen de 10.0 $\mathrm{mL}$ del extracto.

\section{Dopaje de muestras}

A los extractos obtenidos, se les determinó la concentración del $\mathrm{GA}_{3}$ empleando las mejores condiciones cromatográficas determinadas. Posteriormente, y con base en esta concentración, se realizaron dopajes de las muestras. Esto se logró al adicionar 20 mL. del estándar de $\mathrm{GA}_{3}$ a una concentración de $20 \mathrm{mg} / \mathrm{kg}$., de tal forma que la señal del analito aumentara el doble de la señal original [23].

\section{Condiciones cromatográficas}

El análisis del ácido giberélico se realizó en un cromatógrafo líquido de alta eficiencia (HPLC) Shimadzu, equipado con un desgasificador prominence DGU-205, un detector de ultravioleta visible (UV-Vis) con arreglo de diodos (DAD) prominence SPDM20A, un horno Shimadzu CTO-10AS VP y un automuestreador Shimadzu SIL-10AF. El software empleado para el análisis de los datos fue LC-Solution Shimadzu. Se empleó una columna Supelcosil LC-18-DB (150 x 4.6 $\mathrm{mm}$. de diámetro) con un tamaño de partícula de $5.0 \mu \mathrm{m}$.

Para obtener las condiciones óptimas de separación en fase reversa, se inyectaron alternadamente el estándar del GA, muestra dopada y sin dopar, en las diferentes fases 
móviles y flujos empleados por HPLCDAD. Posterior a la elección de las mejores condiciones para el eluyente, se varió la longitud de onda para seleccionar la de mejor absorción. El flujo óptimo se determinó a partir de la curva de Van Deemter, la cual se obtuvo empleando los datos de tiempo de retención y ancho del pico del estándar del

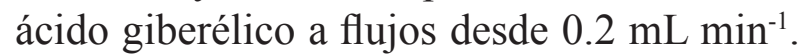
hasta $0.8 \mathrm{~mL} \mathrm{~min}^{-1}$. Para determinar la fase móvil óptima, se realizaron pruebas basadas en los trabajos de Bhalla et al., [13], Xie et al., [21] y Stinemetz y Roberts [6], los cuales realizaron corridas cromatográficas en modo isocrático, empleando mezclas de acetonitrilo-ácido fórmico $0.01 \%$ y metanol-

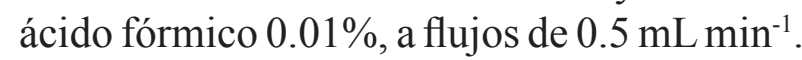
y $0.6 \mathrm{~mL}$ min-1. respectivamente. Finalmente, se realizaron corridas en gradiente empleando los mismos solventes y flujos.

Para identificar la presencia del ácido giberélico en las muestras se realizaron comparaciones entre el tiempo de retención de la muestra y del estándar, se hicieron dopajes de la muestra, se determinó la pureza espectral de pico y se realizaron análisis por cromatografía líquida de alta eficiencia acoplada a espectrometría de masas (HPLCMS). Estos análisis se realizaron en un equipo de cromatografía líquida de alta eficiencia Elite LaChrom (VWR - HITACHI) con detector de arreglo de diodos y acoplado a un espectrómetro de masas Amazon $\mathrm{X}$ (BrukerDaltonis) con ionización por electrospray (ESI) y analizador de trampa iónica (IT). El detector espectrométrico de masas se operó en modo Full scan negativo.

\section{Análisis de datos}

Se realizó un análisis descriptivo de los datos en el cual se tomaron medidas de tendencia central, medidas de dispersión y análisis de regresión de Van Deemter. Todos los experimentos realizados se efectuaron por triplicado y de forma aleatorio.

\section{RESULTADOS}

\section{Optimización de las condiciones cromatográficas}

Se ensayaron varios tipos de fases móviles isocráticas, en las que se mezclaron metanol, acetonitrilo y agua para conseguir una separación adecuada del ácido giberélico. Bhalla et al., [13] y Xie et al., [21], ya habían estudiado un tipo similar de fases móviles para separar el $\mathrm{GA}_{3}$ en muestras vegetales, logrando buena separación y obteniendo el analíto en estudio con tiempo de retención de $3.651 \mathrm{~min}$. Sin embargo, en este estudio no se logró una apropiada separación del $\mathrm{GA}_{3}$ bajo estas condiciones, esto tal vez debido a la complejidad de la matriz de estudio; evidenciada en el amplio intervalo de compuestos con diferente polaridad (Figura 1); por lo que fue necesario evaluar fases móviles en gradiente.

Los mejores resultados en la separación del analito se obtuvieron trabajando con una fase móvil compuesta por una solución de acetonitrilo-ácido fórmico $0.01 \%$ en elución en gradiente con aumento lineal en el tiempo, iniciando con $10 \%$ de acetonitrilo por $5 \mathrm{~min}$., incrementándolo hasta $70 \%$ en $25.0 \mathrm{~min}$. y manteniéndolo durante $15.0 \mathrm{~min}$ (Figura 1). Por último, se disminuyó la proporción del acetonitrilo hasta $10 \%$ durante $5.0 \mathrm{~min}$. obteniéndose una corrida de $45.0 \mathrm{~min}$. Wu et al., [7] y Xie et al., [21] en una separación de giberelinas extraídas de vegetales y frutas, evaluaron diferentes solventes y eligieron agua y acetonitrilo como fases móviles por la buena resolución y separación obtenida. Al igual que los resultados obtenidos en este trabajo, Wu et al., [7] iniciaron con alto porcentaje de agua, aumentando el porcentaje total de acetonitrilo a lo largo de la separación cromatográfica. Bajo estas condiciones se encontró al patrón de ácido giberélico $\left(\mathrm{GA}_{3}\right)$ a una longitud de onda máxima de absorción de $195 \mathrm{~nm}$; por debajo de lo reportado 
por Macías et al., [14], Bhalla et al., [13] y Schneider et al., [24], quienes obtuvieron la mejor señal a 205 nm., 206 nm. y $210 \mathrm{~nm}$. respectivamente.

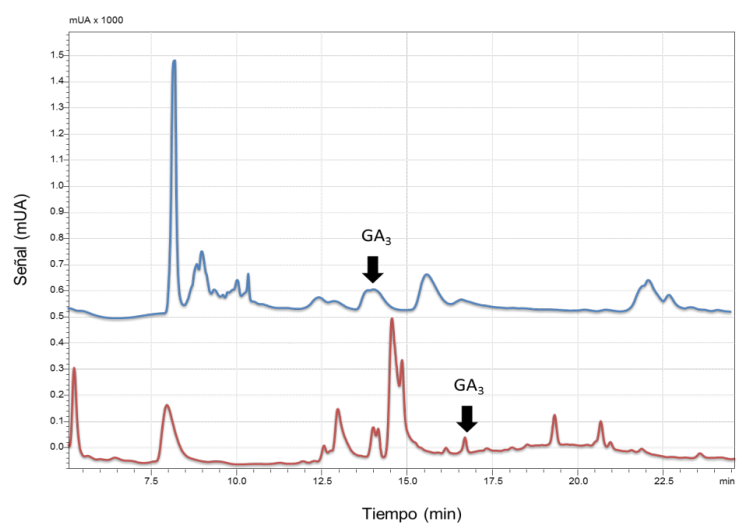

Figura 1. Cromatogramas de muestra de semillas de maíz: elución isocrática (azul), elución en gradiente (rojo)

La curva de Van Deemter (Figura 2) muestra la relación entre la velocidad lineal (u) de la fase móvil y la altura equivalente de plato teórico (HETP). En esta se observa que la menor altura de equivalente de plato teórico $(0.0025 \mathrm{~mm}$.) se obtuvo cuando la velocidad del flujo fue de $0.048 \mathrm{~cm} \mathrm{~s}^{-1}$., por lo tanto, a esta velocidad se logró la mejor eficiencia de la columna, lo cual es ideal en procesos cromatográficos [25]. La velocidad lineal de $0.048 \mathrm{~cm} \mathrm{~s}^{-1}$. corresponde a un flujo de 0.5 $\mathrm{mL} \mathrm{min}^{-1}$, por consiguiente, este flujo fue el óptimo y se empleó para el análisis del ácido giberélico en este estudio.

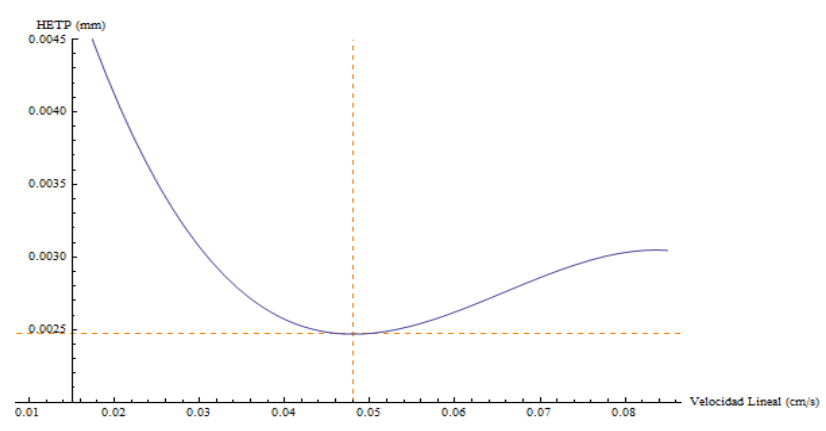

Figura 2. Curva de Van Deemter para el ácido giberélico
Por tanto, las condiciones óptimas para el análisis del ácido giberélico por HPLC son: fase móvil en elución en gradiente con aumento lineal en el tiempo de acetonitriloácido fórmico $0.01 \%$, flujo de $0.5 \mathrm{~mL} \mathrm{~min}$ 1. y longitud de onda de $195 \mathrm{~nm}$. Además, la temperatura implementada fue de $30^{\circ} \mathrm{C}$. Bajo estas condiciones cromatográficas el ácido giberélico eluyó a un tiempo de retención $\left(t_{R}\right)$ de $16.670 \pm 0.050$ min. (Figura 3). Estas condiciones cromatográficas son semejante a las trabajadas por Urbanova et al., 2013 en plantas de Arabidopsis thaliana y por $\mathrm{Wu}$ et al., 2012 en plantas de arroz (oryza sativa) quienes reportaron análisis en fase reversa con tiempos de corrida $30.0 \mathrm{~min}$. y tR para el $\mathrm{GA}_{3}$ de 6.8 min. y 9.7 min. respectivamente.

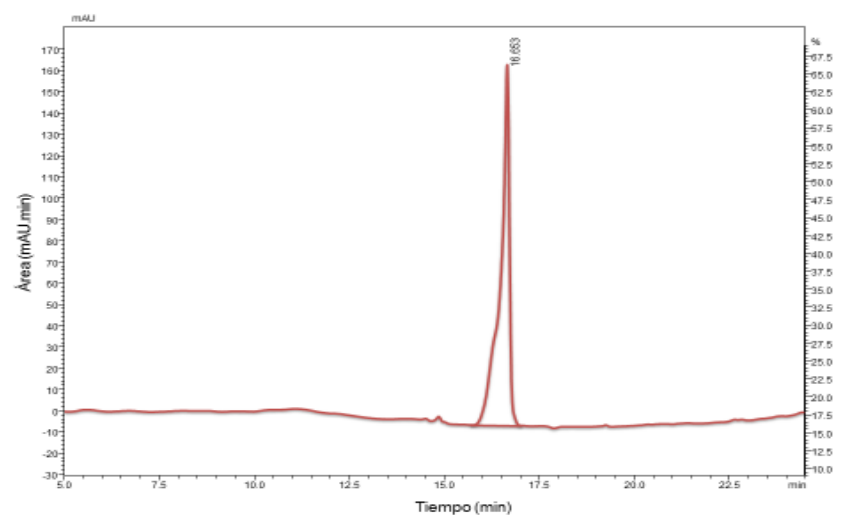

Figura 3. Cromatograma del ácido giberélico $50 \mathrm{mg} \mathrm{kg}^{-1}$.

\section{Identificación del ácido giberelico en muestras}

En la Figura 4 se pueden observar los cromatogramas superpuestos de un estándar delácido giberélico y de una muestra específica eluídos bajo las condiciones cromatográficas seleccionadas como ideales. Se puede observar que el estándar (azul) presentó un tiempo de retención de 16.680 min., mientras que en la muestra (rojo) se observó un pico a 16.653 min., estos tiempos tan cercanos 
fueron el primer indicativo de que la muestra contenía ácido giberélico. Al verificar la pureza espectral del pico en la muestra, se encontró un valor del $99.18 \%$, lo cual indica que es un pico puro, en el cual los excipientes de la matriz no generaron señales que pudieran interferir con la señal del analito. La pureza de pico debe ser mínimo del $99.00 \%$ para considerar que no hay presencia de impurezas [26]. Además, también se compararon los espectros de absorción tomados en la porción ascendente, máxima y descendente del pico correspondiente al estándar de $\mathrm{GA}_{3}$ y al de esta fitohormona en la muestra, siendo estos iguales y demostrando la especificidad del método para la molécula en estudio. Gómez et al., [27], en un trabajo con HPLC, realizaron la comparación entre el espectro del pico del estándar de ibuprofeno y el espectro de la solución muestra, y determinaron la pureza de pico para la identificación de la molécula en estudio, demostrando que había una correlación espectral del 99.5\%.

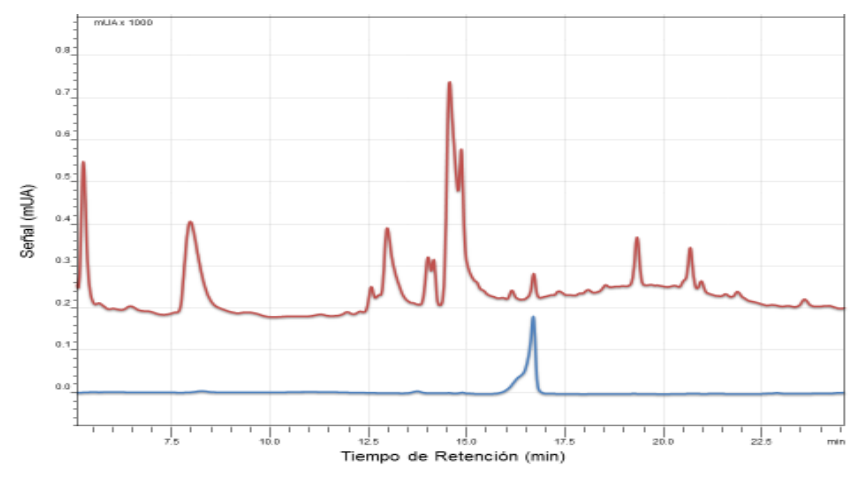

Figura 4. Cromatogramas de $\mathrm{GA}_{3}$ (azul) y muestra de semillas de maíz (rojo)

En la Figura 5 se pueden observar los cromatogramas de los extractos de semillas de maíz germinadas sin dopaje (rojo) y los extractos de la muestra dopada (azul). Encontrando a un tiempo de retención de $16.668 \mathrm{~min}$. un aumento en la muestra dopada, lo que permitió inferir que esta señal efectivamente corresponde a la del analito de interés $\left(\mathrm{GA}_{3}\right)$, además la pureza de pico de la muestra dopada no varía con respecto a la muestra sin dopar.

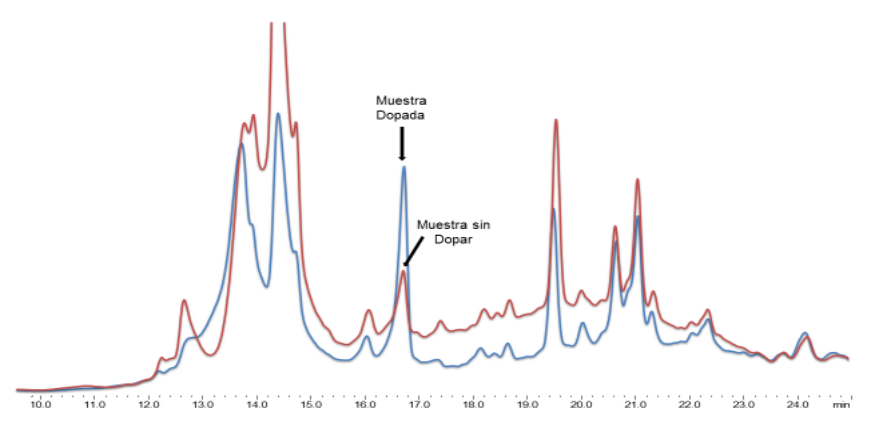

Figura 5. Cromatograma de muestra dopada (azul) y sin dopar (rojo)

Cromatografía líquida de alta eficiencia acoplada a espectrometría de masas (HPLCMS) Mediante HPLC-MS se pudo determinar el patrón de fragmentación característica del $\mathrm{GA}_{3}$ (Figura 6) al detectar el ion molecular a $345.0 \mathrm{~m} / \mathrm{z}$, el pico base a $239.1 \mathrm{~m} / \mathrm{z}$ correspondiente a rupturas heterolíticas del grupo carboxilo en el carbono 6, de grupos hidroxilos en el carbono 3 y 13 y del grupo metilo del carbono 4. Además se evidencian los iones característicos a Cromatografía líquida de alta eficiencia acoplada a espectrometría de masas (HPLC-MS) Mediante HPLC-MS se pudo determinar el patrón de fragmentación característica del GA (Figura 6) al detectar el ion molecular a $345.0 \mathrm{~m} / \mathrm{z}$, el pico base a 239.1 $\mathrm{m} / \mathrm{z}$ correspondiente a rupturas heterolíticas del grupo carboxilo en el carbono 6, de grupos hidroxilos en el carbono 3 y 13 y del grupo metilo del carbono 4. Además se evidencian los iones característicos a $143.5 \mathrm{~m} / \mathrm{z}$ y 221.2 $\mathrm{m} / \mathrm{z}$ correspondientes a la ruptura heterolítica del anillo de lactona y de los anillos C y D respectivamente (Figura 7). Estos datos concuerdan con lo reportado por Xie et al., [21] y Ge et al., [17], lo cual permite inferir que se está obteniendo exclusivamente $\mathrm{GA}_{3}$ 


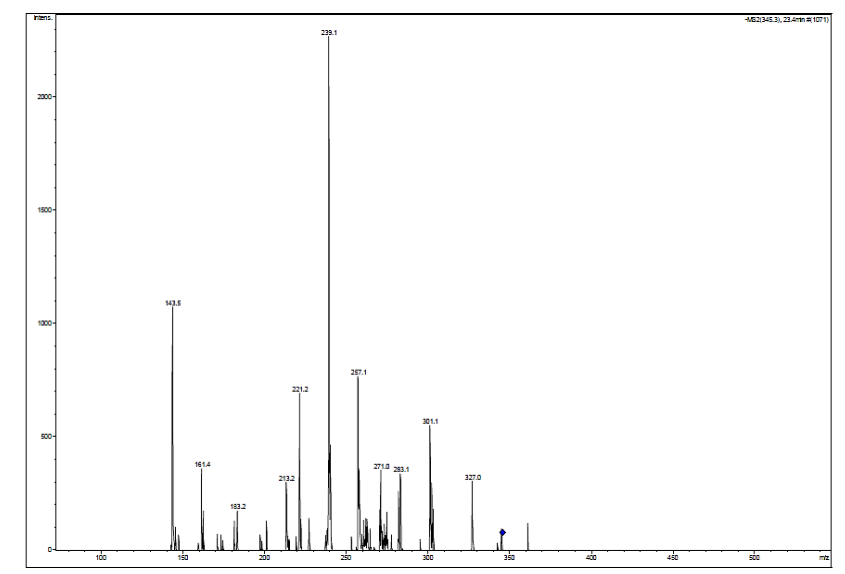

Figura 6. Espectro de masas del pico del ácido giberélico en una muestra con sus respectivos iones característicos.

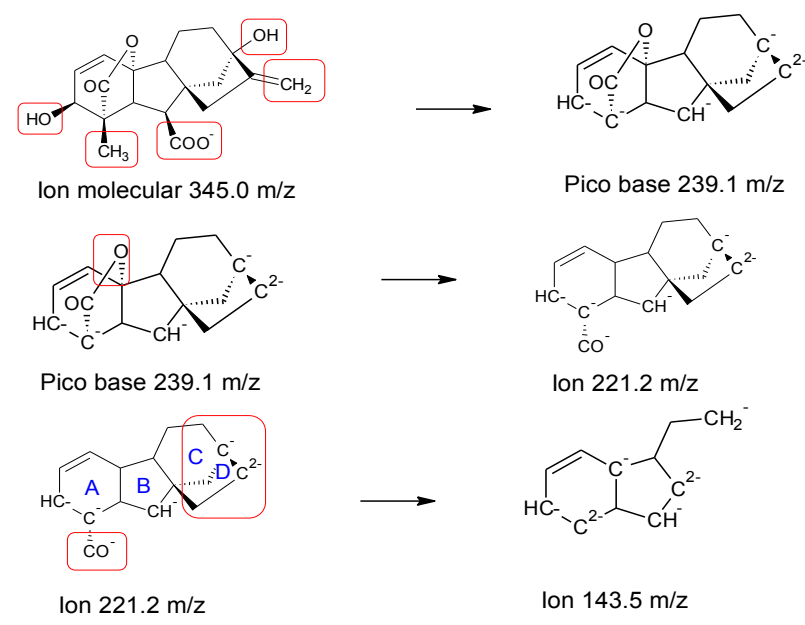

Figura 7. Iones característicos del ácido giberélico elaborado por el autor.

Estos resultados permitieron concluir que la metodología es óptima para separar y detectar de manera inequívoca el ácido giberélico en semillas germinadas maíz. Lo anterior facilitará que al estudiar semillas de diferente tipo y procedencia, se pueda determinar la calidad de las mismas mediante la realización de un tamizaje inicial en el que se cuantificará esta fitohormona y de esta manera se puedan emplear metodologías como los campos magnéticos que estimularán el nivel de $\mathrm{GA}_{3}$ lo que daría como resultado una germinación en más corto tiempo.

\section{CONCLUSIONES}

A pesar de trabajar una matriz compleja, el método cromatográfico propuesto en este artículo permite distinguir, de manera simple, rápida y reproducible, entre el ácido giberélico y otros compuestos no identificados. Por tanto, es seguro utilizarlo en estudios posteriores, e incluso podría adaptarse para la determinación de esta molécula en otras matrices. Lo cual lo hace potencial para su implementación en laboratorios interesados en análisis de fitohormonas.

\section{AGRADECIMIENTOS}

A la Vicerrectoría de Investigaciones y Posgrados de la Universidad de Caldas por la financiación del proyecto con código 0455216.

\section{BIBLIOGRAFÍA}

[1] ASTURIAS, M. A., Maíz de alimento sagrado a negocio del hambre. Quito, 2004.

[2] FAO, El estado mundial de la agrícultura y la alimentación, Roma, 1993, 26.

[3] PÉREZ F., "Germinación y Dormición de Semillas," in Material Vegetal de Reproduccion; Manejo, Conservacion y Tratamiento, España: Consejeria de Medio Ambiente, 2003, pp. 177-198.

[4] AZCON-BIETO, J.; TALÓN, M., Fundamentos de fisiología Vegetal. Barcelona: McGraw-Hill Interamericana, 2008.

[5] URBANOVÁ, T.; TARKOWSKÁ, D.; NOVÁK, O.; HEDDEN, P.; STRNAD, M., "Analysis of gibberellins as free acids by ultra performance liquid chromatography-tandem mass spectrometry.," Talanta, 2013, 112, pp. 85-94.

[6] STINEMETZ, C. L.; ROBERTS, B. R.; "An Analysis of the Gibberellic and 
Abscisic," J. Arboric., 1984, 10(10) pp. 283-285.

[7] WU, Q.; WU, D.; DUAN, C.; SHEN, Z.; GUAN, Y., "Hollow fiber-based liquidliquid-liquid micro-extraction with osmosis: II. Application to quantification of endogenous gibberellins in rice plant," J. Chromatogr. A, 2012, 1265, pp. 17-23.

[8] SORIANO, M. D.; BOTTINI, R., "Gibberellin A3 levels in bark and flower buds of peach (Prunus persica L.) before leaf fall," Agriscientia, 1992, IX(2), pp. 3-8.

[9] White, C. N.; PROEBSting, W. M.; HEDDEN, P.; RIVIN, C. J., "Gibberellins and Seed Development in Maize. I. Evidence That Gibberellin/Abscisic Acid Balance Governs Germination versus Maturation Pathways," Plant Physiol., 2000, 122, pp. 1081-1088.

[10] RIVERA, J. D.; CORREA, Y. M.; PENAGOS, J.P., "Evaluación de métodos de extracción para la obtención del ácido giberélico en semillas germinadas de maíz (Zea mays L.)," Rev. Colomb. Química, 2017, 46(2), pp. 45-50.

[11] TAIZ, L.; Zeiger, E., Plant Physiology, Third Edit. Sunderland: Sinauer Associates, 2002.

[12] SACKETT, P. H.; "High-Performance Thin-Layer Chromatography of Gibberellins in Fermentation Broths," Anal. Chem. ACS, 1984, 56(9), pp. 1600-1603.

[13] BHALLA, K.; SINGH, S. B.; Agarwal, R., "Quantitative determination of gibberellins by high performance liquid chromatography from various gibberellins producing Fusarium strains," Environ. Monit. Assess., 2010, 167, pp. 515-520.
[14] MACÍAS, J. M.; POURNAVAB, R. F.; REYES-VALDÉS, M. H.; BENAVIDES-MENDOZA, A., "Development of a Rapid and Efficient Liquid Chromatography Method for Determination of Gibberellin A4 in Plant Tissue, with Solid Phase Extraction for Purification and Quantification," Am. J. Plant Sci., 2014, 5, pp. 573-583.

[15] MANZI, M.; GÓMEZ, A.; ARBONA, V., "Rapid and reproducible determination of active gibberellins in citrus tissues by UPLC / ESI-MS / MS," Plant Physiol. Biochem., 2015, 94, pp. 1-9.

[16] URBANOVÁ, T.; TARKOWSKÁ, D.; STRNAD, M.; HEDDEN, P., "Gibberellins - terpenoid plant hormones: Biological importance and chemical analysis," Collect. Czechoslov. Chem. Commun., 2011, 76(12), pp. 1669-1686.

[17] GE, L.; PEH, C. Y. C.; YONG, J. W. H.; TAN, S. N.; HUA, L.; ONG, E. S., "Analyses of gibberellins by capillary electrophoresis-mass spectrometry combined with solid-phase extraction," J. Chromatogr. A, 2007, 1159, pp. 242249.

[18] SHARP, R. G.; ELSE, M. A.; DAVIES, W. J.; CAMERON, R. W., "Gibberellinmediated suppression of floral initiation in the long-day plant Rhododendron cv. Hatsugiri," Sci. Hortic. (Amsterdam)., 2010, 124, pp. 231-238.

[19] KANG, S. M.; KIM, J. T.; HAMAYUN, M.; HWANG, I. C.; KHAN, A. L.; KIM, Y. H.; LEE, J. H.; LEE, I. J., "Influence of prohexadione-calcium on growth and gibberellins content of Chinese cabbage grown in alpine region of South Korea," Sci. Hortic. (Amsterdam)., 2010, 125, pp. 88-92. 
[20] HAMAYUN, M.; KHAN, S. A.; KHAN, A. L.; AHMAD, N.; NAWAZ, Y.; SHER, H.; LEE, I. J., "Gibberellin producing Neosartorya sp. CC8 reprograms Chinese cabbage to higher growth," Sci. Hortic. (Amsterdam)., 2011, 129, pp. 347-352.

[22] CORREA, Y. M.; BURITICÁ, L. M.; RIVERA, J. D.; PENAGOS, J. P.; TORRES, J. I., "Optimización del protocolo para la extracción y la cuantificación de proteínas totales en semillas germinadas de maíz (Zea mays L.)," Rev. Fac. Ciencias Basicas, 2017, 13(1), pp. 60-64.

[23] HARRIS, D. C., Quantitative Chemical Analysis, Eighth Edi. New York: W. H. Freeman and Company, 2010.
[23] HARRIS, D. C., Quantitative Chemical Analysis, Eighth Edi. New York: W. H. Freeman and Company, 2010.

[25] MEYER, V. R., Practical HighPerformance Liquid Chromatography, Fifth Edit. New York: A John and Sons, Ltd, 2010.

[26] DE ZAN, M. M., "Utilización de quimiometría para mejorar el rendimiento de la cromatografía líquida de alta resolución," Universidad Nacional del Litoral, 2011.

[27] GÓMEZ, S. M.; MARTÍNEZ, J. A.; MARTÍNEZ, F., "Validación de un método analítico empleando cromatografía líquida de alta eficiencia para la determinación de ibuprofeno en medios biorrelevantes," Quim. Nova, 2000, 33(8), pp. 1794-1799. 